\title{
Scalable HetNet interference management and the impact of limited channel state information
}

\author{
Alessandro Chiumento ${ }^{1,2^{*}}$, Sofie Pollin ${ }^{1,2}$, Claude Desset ${ }^{1}$, Liesbet Van der Perre ${ }^{1,2}$ and Rudy Lauwereins ${ }^{1,2}$
}

\begin{abstract}
Interference management is important in wireless cellular networks such as long-term evolution (LTE) and LTE-A, where orthogonal frequency division multiple access (OFDMA), dense frequency reuse and heterogeneous cell sizes and capabilities provide great performance at the cost of increased network complexity. The layered structures of emerging cellular networks and their dynamic environments limit greatly the efficacy of traditional static interference management methods. Furthermore, conventional interference coordination techniques assume that perfect channel knowledge is available and that the signalling overhead can be neglected. In this paper, we analyse a heterogeneous LTE OFDMA downlink network composed by macro-, pico- and femtocells. We propose a low-complexity, distributed and cooperative interference mitigation method which is aware of network load and propagation conditions. The proposed method is fully scalable and addresses the interference received by the macro and pico layer and the interference received by femtocells separately. The new solution makes use of the iterative Hungarian algorithm, which effectively reduces interference and enhances the quality of service of starved users when compared to other state-of-the-art solutions. The proposed method outperforms static solutions by providing comparable results for the cell edge users (the proposed solution delivers $86 \%$ of the gain of a static frequency reuse 3 ) while presenting no loss at the cell centre, compared to an 18\% loss of the frequency reuse 3 in a homogeneous scenario. In a heterogeneous network (HetNet) deployment, it generates a gain of $45 \%$ for the combined macro and pico edge users at a very small cost for the cell centre lower than $4 \%$ when compared with standard resource allocation. It optimizes greatly picocell performance, with improvements of more than $50 \%$ at a small cost for femtocell users (15\%). In order to apply the proposed method to a practical network, the impact of the necessary quantization of channel state information on the interference management solution is studied and results show that signalling overhead can be contained while performance is improved by increasing resolution on the portions of the bandwidth more likely to be assigned to the users.
\end{abstract}

Keywords: LTE; LTE-A; Heterogeneous network; Interference coordination; Limited CSI

\section{Introduction}

The rapid increase in mobile devices in recent years has pushed the mobile service providers to implement new technologies to enhance the network's capacity and provide a faster, more reliable service. The 'de-facto' standard for current generation of high-speed cellular communication networks is the long-term evolution (LTE) system [1].

\footnotetext{
${ }^{*}$ Correspondence: alessandro.chiumento@esat.kuleuven.be

1 Interuniversity Micro-Electronics Center (IMEC) vzw, Kapeldreef-75, B-3001 Leuven, Belgium

${ }^{2}$ Department of Electrical Engineering (ESAT) KU Leuven, Kasteelpark Arenberg 10, B-3001 Leuven, Belgium
}

A key feature of LTE is the use of orthogonal frequency division multiple access (OFDMA). OFDMA was chosen as LTE's downlink access scheme as its flexibility allows the operator to allocate resources to each user independently, increasing the system's flexibility to exploit channel and multi-user diversity. On the other hand, the need for capacity growth has brought operators to increase the network's density and to push for a high-frequency reuse factor. Inter-cell interference (ICI) is then a problem that has to be addressed in order to provide the user with the quality of service (QoS) necessary for a reliable communication.

Traditional inter-cell interference coordination (ICIC) strategies for homogeneous networks include careful

\section{算 Springer}

(c) 2015 Chiumento et al: licensee Springer. This is an Open Access article distributed under the terms of the Creative Commons Attribution License (http://creativecommons.org/licenses/by/4.0), which permits unrestricted use, distribution, and reproduction in any medium, provided the original work is properly credited. 
planning, spectrum splitting or power control. One of the typical techniques found in literature to reduce the impact of ICI is frequency reuse (FR) [2]: the available frequency band in the cell is split into one part for 'centre users' and an other one for 'edge users'. This frequency assignment can either be static or dynamic; static techniques, such as Soft FR [3] and Fractional FR [4] offer good interference mitigation but at the cost of a limited spectral efficiency. These static techniques do not take into consideration the load of the network or channel conditions; the spectrum is assigned statically to edge users also when the bandwidth could be used by cell centre users. Central users have favourable channel conditions; they are bandwidth limited and not interference limited so allocating part of their spectrum to edge users will limit their data rate. Dynamic techniques, on the other hand, can adapt to the network's operating conditions and do not require any prior frequency planning. In [5], an adaptive self-organizing FR scheme is proposed. It delivers interesting results yet it relies largely on offline simulations to achieve a solution space, making it impractical in scenarios where the network might change drastically and unpredictably, e.g., in heterogeneous networks or servicing high-mobility users. In [6], a dynamic, distributed resource allocation algorithm is presented, where users are allocated only on high-gain subchannels. Subsequently, neighbouring cells communicate their edge users' resource allocation to each other in order to minimize interference. Then, power control is applied onto the assigned subchannels. The method provides increased cell edge performance without, however, analysing the degradation at the cell's centre. Typically, techniques that improve cell edge have large penalty on cell centre, so it should be studied. Wang et al. in [7] introduce a centralized dynamic fractional frequency reuse scheme which shows an improved behaviour for edge users but makes use of a centralized controller. Rahman et al. presented two ICIC (ICI coordination) schemes based on the iterative Hungarian algorithm (IHA). In [8], they introduce a distributed mechanism in which neighbouring base stations communicate to coordinate ICI limitation. In this work, the overall propagation conditions of the network are not considered and ICIC is allowed only between pairs of neighbouring sectors. In [9], they extend their previous work by introducing a centralized controller which improves ICIC over [8].

The introduction of small cells, such as pico- and femtocells, has further increased the complexity of ICI management. Picocells (PeNB) are managed by the network operator and share spectrum and resources with the larger macrocells (eNB). These small cells are, generally, carefully placed to compensate for heavily congested areas or for poor coverage, e.g. indoors. Picocells share a backhaul connection with macrocells and can thus communicate with each other and allow for synchronization and coordination. Femtocells (HeNB), on the other hand, are small cells owned and managed by private customers and are generally designed to be indoor access points [10]. Communication between macro- (pico-) and femtocells is not envisioned, and hence, coordination between the two layers is impossible [11]. In order to mitigate interference from femto base stations, different strategies have been presented. One way would be to utilize orthogonal spectrum for the two layers, in a way similar to fractional FR, at the cost of spectral efficiency [12]. To maximize spectral efficiency, then macro and femto base stations have to work in shared spectrum (or co-channel deployment), interfering with each other. Kosta et al. [13], Kaimaletu et al. [14] contain surveys of the most common ICIC techniques used in literature.

The vast majority of ICIC techniques makes the assumption that the users are able to determine their channel conditions for the whole bandwidth with a high resolution. Furthermore, each user is supposed to differentiate between the interfering base stations. In a practical system, however, there is a limit in channel state information (CSI) estimation accuracy as well as feedback bandwidth availability. The users are only able to report CSI information on a limited subset of the overall bandwidth. They might not be able to identify the highest interferer and this interferer's power, on the complete bandwidth, as assumed by traditional ICIC schemes.

In this work, we present a simple, cooperative, distributed interference management scheme which addresses interference problems experienced and generated by macro, pico, and femto base stations. The principles used to provide ICIC are identical for all three cell types; however, the method adapts to the different base station capabilities. Each base station uses the channel state information from its serving users to obtain knowledge on the interfering cells; then, this information is exchanged. In order to minimize interference, macro base stations are able to restrict transmission on portions of the bandwidth if this would maximize the overall network capacity. The procedure follows similarly for picocells, the only difference is that, since picocells are much smaller, they allow for power control. Instead of limiting transmission on whole portions of the bandwidth, a water-filling power control is applied to reduce interference, according to [15]. The cooperative nature of the proposed method,allows each base station to limit interference to the neighbours in a way that optimizes the overall network's conditions. As femtocells are unable to communicate with other base stations, in this work, it is assumed that they exploit spectrum sensing in order to detect macro communications; they are able to sense the environment and overhear uplink communication between a macrocell user and its serving base station if 
such user is close enough to the femtocell. Cognitive femtocells can make use of advanced sensing technologies such as presented in [16] and are currently studied as potential solutions for the small cell coordination problem $[17,18]$.

Furthermore, in [19], we have shown that it is possible to limit the amount of CSI feedback adaptively when too detailed information is not beneficial for overall system performance, i.e. the cost of feedback is higher than the benefit. Hence, the second contribution of this work is to extend the ICIC method here presented to a practical system where the channel state information can be reduced. We analyse the impact of this quantization on the system and suggest a solution to enhance performance.

This paper is further organized as follows. In Section 2 , we introduce the system model and describe the base station capabilities. In Section 3, the proposed algorithms are presented and discussed, and in Section 4, we show the improved results for the proposed method based on simulations when highest CSI is assumed. In Section 5, we discuss the effects of channel state information quality on ICIC and the uplink feedback. In Section 6, the conclusions are summarized.

\section{System model of LTE-A downlink network}

We consider a heterogeneous LTE downlink OFDMA network composed of $C$ macro base stations (eNBs). Each macro base station is composed by $S$ orthogonal sectors; each sector is responsible to serve a portion of the overall cell area. There are, thus, a total of $M=C \cdot S$ macrocell sectors in the network. We also simulate $P$ picocells and $F$ femtocells. These small cells contain only one sector each.

Each macrocell's sector serves $X_{m}$ terminals while each pico and femto base station serve $X_{p}$ and $X_{f}$ users, respectively. $K$ physical resource blocks (RBs) can be allocated per sector. The number of RBs is dependent on the cell's bandwidth and can vary between $6(1.4 \mathrm{MHz}$ bandwidth) and 100 (20 MHz bandwidth). In order to simulate the environment, a modified version of the LTE system level simulator developed by Ikuno et al. [20] is used. Each terminal possesses, initially, channel state knowledge between itself and each of the interfering base stations on each RB. This resolution can then be decreased to study the effects of CSI quantization on the resource allocation and interference management. When each base station transmits on all possible resource blocks, the signal-tonoise-and-interference ratio (SINR) at the user's terminal $x_{i}$, on RB $k$, is given by:

$$
\gamma_{x_{i}, k}=\frac{P_{x_{i}, k}^{i} \cdot G_{x_{i}, k}^{i}}{\sigma^{2}+\sum_{\substack{j=1 \\ j \neq i}}^{i} P_{x_{i}, k}^{j} \cdot G_{x_{i}, k}^{j}},
$$

where $P_{x_{i}, k}^{i}$ represents the power transmitted by base station $i$ to its served terminal $x_{i}$ on RB $k$ and $G_{x_{i}, k}^{i}$ is the corresponding channel gain. $P_{x_{i}, k}^{j}$ and $G_{x_{i}, k}^{j}$ are the powers transmitted and the gains from the other base stations, $j \neq i$, which are interfering to the user $x_{i}$ on resource block $k . \sigma^{2}$ is the noise power at the terminal. According to [21], the SINR measured by the mobile user is mapped onto respective channel quality indicator (CQI). Each CQI represents the highest possible modulation and coding scheme (MCS) the terminal can process with a block error rate lower than $10 \%$. This MCS defines then the instantaneous throughput the user would achieve per RB. Table 1 shows this mapping.

The users then exchange this information with the base stations as CSI. Each sector is designed to have a welldefined coverage region as depicted in Figure 1a. While it is reasonable to assume that the closest neighbours contribute to the largest part of the interference, it is also important to notice that when phenomena like shadowing and channel fading are considered, the geometry of the network varies drastically. Figure $1 \mathrm{~b}$ shows how the sector coverage areas may be affected. Hence, the concept of neighbour needs to be redefined, not as a spatially close sector but as a sector that influences substantially the SINR.

The instantaneous data rate $r_{x_{i}, k}$ for user $x_{i}$ on RB $k$ can be directly determined from the SINR $\gamma_{x_{i}, k}$. As the network operator has control of the macro and pico nodes, the objective of the resource allocator is then to maximize the overall rate by minimizing the interference received and generated by these nodes. In order to allocate

Table 1 SINR and CQI mapping to modulation and coding rate at full load

\begin{tabular}{lcccc}
\hline SINR & CQI & Modulation & $\begin{array}{c}\text { Code rate } \\
(\times \mathbf{1}, \mathbf{0 2 4})\end{array}$ & $\begin{array}{c}\text { Spectral } \\
\text { efficiency }\end{array}$ \\
\hline-6.93 & 1 & QPSK & 78 & 0.1523 \\
-5.14 & 2 & QPSK & 120 & 0.2344 \\
-3.18 & 3 & QPSK & 193 & 0.3770 \\
-1.25 & 4 & QPSK & 308 & 0.6016 \\
0.76 & 5 & QPSK & 449 & 0.8770 \\
2.69 & 6 & QPSK & 602 & 1.1758 \\
4.69 & 7 & 16QAM & 378 & 1.4766 \\
6.52 & 8 & 16QAM & 490 & 1.9141 \\
8.57 & 9 & 16QAM & 616 & 2.4063 \\
10.36 & 10 & 64QAM & 466 & 2.7305 \\
12.28 & 11 & 64QAM & 567 & 3.3223 \\
14.17 & 12 & 64QAM & 666 & 3.9023 \\
15.88 & 13 & 64QAM & 772 & 4.5234 \\
17.81 & 14 & 64QAM & 873 & 5.1152 \\
19.82 & 15 & 64QAM & 948 & 5.5547 \\
\hline
\end{tabular}




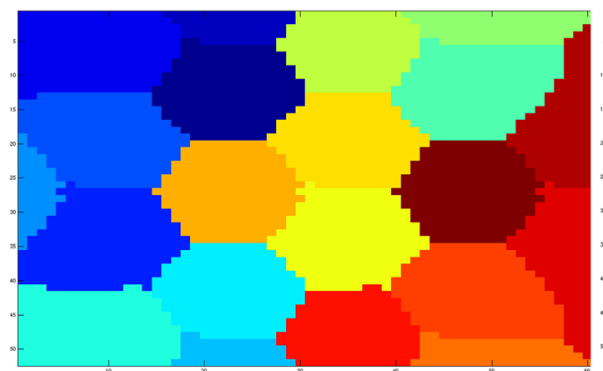

(a) Sectors coverage areas

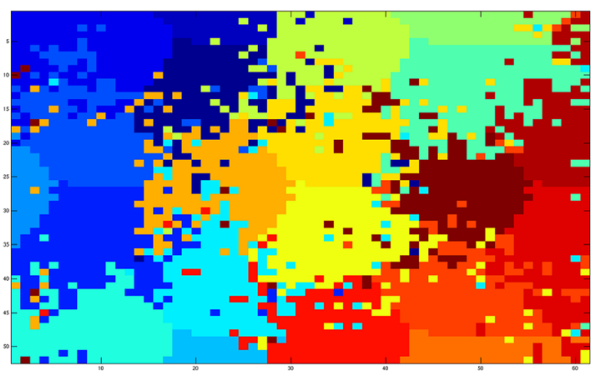

(b) Sectors coverage areas with $10 \mathrm{~dB}$ shadowing and fading

Figure 1 Coverage areas. (a) Well-defined coverage region. (b) How the sector coverage areas may be affected.

resources optimally, the total sum rate of the network has to be maximized, according to:

$$
\max \sum_{i}^{M+P+F} \sum_{x_{i}}^{X_{i}} \sum_{k}^{K} r_{x_{i}, k} \cdot d_{x_{i}} \cdot a_{x_{i}, k}
$$

where

$$
\begin{aligned}
& a_{x_{i}, k}= \begin{cases}1 & \text { when user } x_{i} \text { is assigned on RB } k \\
0 & \text { otherwise }\end{cases} \\
& \sum_{x_{i}}^{X_{i}} a_{x_{i}, k} \leq 1, \forall k \in K, \\
& \sum_{k}^{K} P_{k} \leq P_{\max } \text { for all base stations }
\end{aligned}
$$

$d_{x_{i}}$ is called a demand factor, it is introduced to add some fairness to the allocation; $d_{x_{i}}=\frac{\mu_{x_{i}}}{\bar{R}}$ where $\bar{R}$ is the average rate received by the user over a past time window and $\mu_{x_{i}}$ is the requested rate. The users that receive the lowest rate in the previous transport blocks are then advantaged in the resource allocation process. Constraint (4) makes sure that one RB $k$ might not be assigned to multiple users in a cell. Constraint (5), on the other hand, makes sure that the sum of the power transmitted by a base station on all the resource blocks on each $\mathrm{RB}$ remains below the maximum allowed power; the power in (5) is then the same as in (1). A computational solution for this problem is difficult to find [22]. The Hungarian algorithm (HA) was firstly proposed by Kuhn [23] as a simple solution to binary integer problems under specific conditions, and its iterative version [9] has been studied as a good sub-optimal solution for a generic assignment problem where the number of users and frequency resources can vary dynamically.

Additionally, pico- and femtocells are able to perform power control. While macro base stations can either transmit at full power or not transmit at all on specific RBs, the small cells can reduce power so to minimise interference but still allow for communication on those RBs.

\section{Proposed scalable interference management approach}

The scope of this section is to present a distributed interference management scheme which solves problem (2 to 5), which is not computationally intense and can be applied to practical heterogenous networks (HetNets). The proposed scheme is divided into two parallel parts. The first part deals with interference coming from neighbouring macro or pico base stations while the second part deals with the interference generated by femto base stations.

\subsection{Macro and pico interference management}

The ICI mitigation technique is composed by two phases. Firstly, in the 'restriction definition' phase, each sector determines locally which other sectors are producing harmful interference on specific resource blocks and generates a list of resource blocks that the interfering sectors would have to restrict or limit transmit power in order to improve on that sector's throughput. This is done concurrently with the sector's internal resource scheduling. Secondly, the sectors exchange information with the interfering sectors and in the 'restriction negotiation' phase each sector computes which resource blocks it has to restrict in order to maximize the overall network throughput. The IHA algorithm will be used here to determine which restrictions make sense from a system point of view. Below, the details of the algorithms are explained and summarized in Algorithm 1.

Before the actual introduction of the algorithm, a clarification of the notations used in this work is necessary. Every scalar used in this work is being expressed using a lowercase italic letter, i.e. $x$; an array is presented by a lowercase bold italic letter, such as $\mathbf{l}$ and a matrix is written using a bold capital letter: $\boldsymbol{Y}_{a * b}$ where a and b represent the rows and columns of the matrix, respectively. Each entry of such matrix is expressed with the same letter used to name the matrix, but in lowercase. 


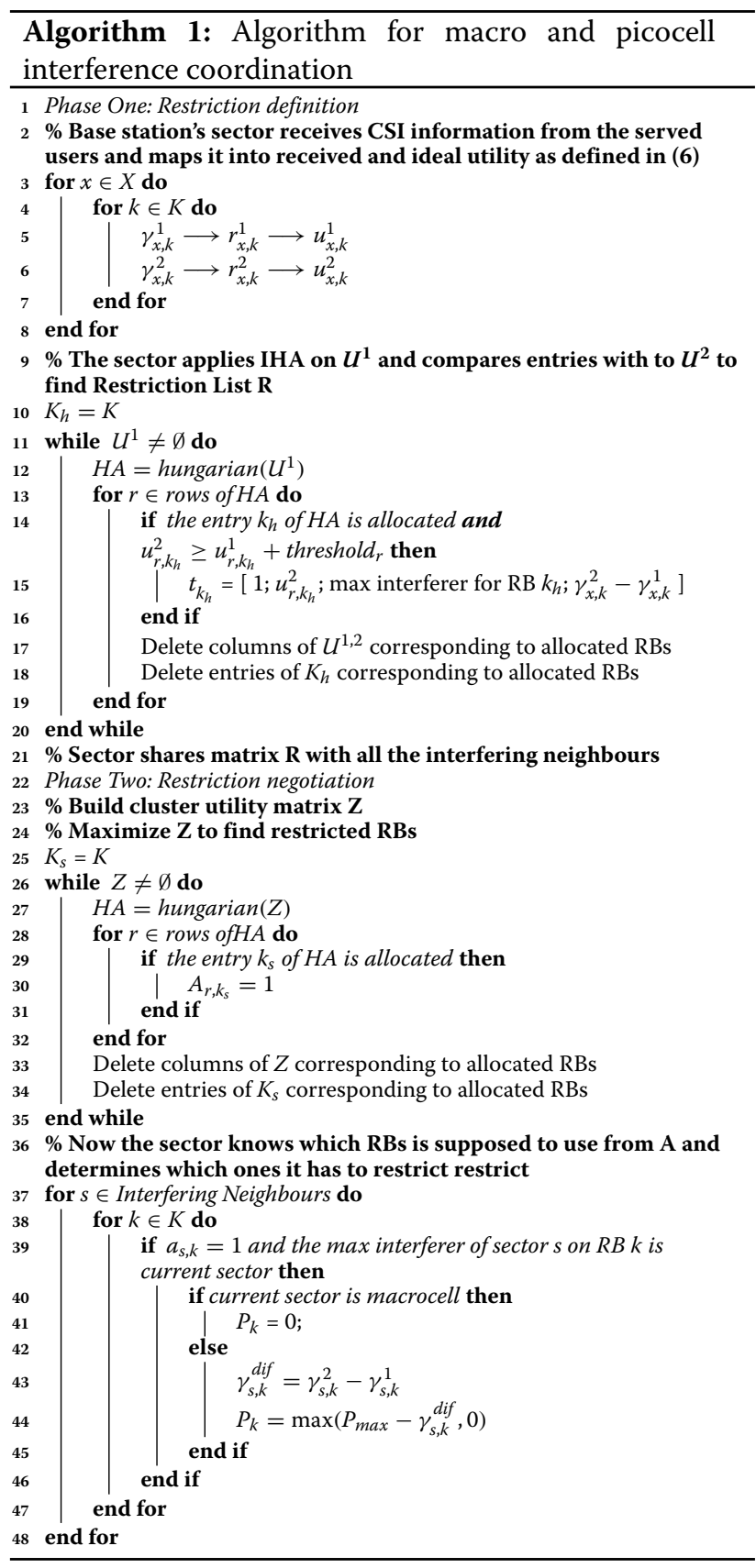

\subsubsection{Restriction definition}

A sector receives the CSI packets containing the CQIs from all the attached users and the power received by each user on each resource block from all the neighbouring base stations. The CSI packets also contain the rate requested by each user and the set of RBs assigned to each user by the base station scheduler. The base station $i$ then, computes the SINR that each user $x_{i}$ experiences on all the resource blocks: $\gamma_{x_{i}}^{1}$ and the SINR $\gamma_{x_{i}}^{2}$ the user would experience if the highest interfering sector on each resource block is forced to zero. These SINR values are then mapped into their relative data rates, $r_{x_{i}}^{1}$ and $r_{x_{i}}^{2}$, for each user $x_{i}$ using the MCS schemes from Table 1 . The rate vectors of each user are then converted into utility vectors $\boldsymbol{u}^{1}$ and $\boldsymbol{u}^{2}$ defined as:

$$
u_{x_{i}, k}^{1}=r_{x_{i}, k}^{1} \cdot d_{x_{i}} \text { and } u_{x_{i}, k}^{2}=r_{x_{i}, k}^{2} \cdot d_{x_{i}}
$$

where $d_{x_{i}}$ is the demand of user $x_{i}$. All the utility vectors $u_{x_{i}}^{1}$ and $u_{x_{i}}^{2}$ are then collected in two utility matrices $\boldsymbol{U}^{1}$ and $\boldsymbol{U}^{2}$ of dimensions $X_{i} \cdot K$, where $X_{i}$ is the number of users served in the cell (either $X_{m}$ or $X_{p}$ ). The restriction choice of a specific resource block $k$ is performed by comparing the utility $u_{x_{i}, k}^{1}$ user $x_{i}$ experiences on RB $k$ with the higher utility $u_{x_{i}, k}^{2}$ and a threshold value $t h_{x_{i}, k}$ :

$$
\text { If } u_{x_{i}, k}^{2} \geq u_{x_{i}, k}^{1}+t h_{x_{i}}
$$

then user $x_{i}$ would see an improved data rate on RB $k$ if the highest interfering sector would be restricted; $t h_{x_{i}}$ can be dynamically adjusted, in this paper, it is equal to the minimal rate requested (see Table 2). If a user is well placed (i.e. in the cell centre), or does not witness interference, the improved utility $\boldsymbol{u}^{2}$ would not be considerably higher than the measured one $\boldsymbol{u}^{1}$. This criterion, then,

\begin{tabular}{|c|c|c|}
\hline Parameters & Symbols & Values \\
\hline Number of macrocells & M & 7 \\
\hline Sectors per macrocell & $S$ & 3 \\
\hline Inter macrocell distance & & $500 \mathrm{~m}$ \\
\hline Macrocell radius & & $150 \mathrm{~m}$ \\
\hline Macro users per sector & $x_{m}$ & 20 \\
\hline Macro transmit Power & & $46 \mathrm{dBm}$ \\
\hline Number of picocells & $P$ & 5 \\
\hline Picocell radius & & $20 \mathrm{~m}$ \\
\hline Pico users per sector & $X_{p}$ & 4 \\
\hline Pico transmit Power & & $20 \mathrm{dBm}$ \\
\hline Number of femtocells & $\mathrm{F}$ & 10 \\
\hline Femtocell radius & & $10 \mathrm{~m}$ \\
\hline Femto users per sector & $X_{f}$ & 2 \\
\hline Femto transmit power & & $10 \mathrm{dBm}$ \\
\hline System bandwidth & & $20 \mathrm{MHz}$ \\
\hline Available resource blocks & & 100 \\
\hline Carrier frequency & & $2.1 \mathrm{Ghz}$ \\
\hline Propagation model & & WINNER II model [24] \\
\hline Internal base station scheduler & & Hungarian scheduler \\
\hline Antenna configuration & & SISO \\
\hline Traffic model & & Full buffer \\
\hline Minimal throughput requested & th & $1 \mathrm{Mbps}$ \\
\hline
\end{tabular}

\section{Table 2 System parameters}


targets users which see an improvement when the interference is reduced, generally speaking these are referred to as 'starved users'.

Not all the resource blocks that fulfill the above criterion will actually be blocked or used at limited power by the neighbours but only the ones that maximize the utility of the sector when all the attached users are scheduled on all the available resource blocks: each sector generates a restriction list $\mathbf{R}$ by applying the iterative Hungarian algorithm onto the utility matrix $\boldsymbol{U}^{1}$. At each iteration $h$, the HA determines which set of RBs $\hat{k}_{h}$ can be assigned to the attached users $X$, as defined in the system model section, so that the utility of the scheduled users for that transport block is maximized. This optimization considers interference, pathloss and shadowing.

If there is improvement in restricting the assigned resource blocks, i.e. if user $x_{i}$ is assigned $\mathrm{RB} \hat{k_{h, x_{i}}}$ at iteration $h$ and $u_{x_{i}, \hat{k_{h} i}}^{2} \geq u_{x_{i}, k_{h, x_{i}}}^{1}+\mathrm{th}_{x_{i}}$ then the corresponding entry of the restriction list $\mathbf{R}, r_{k_{h, x_{i}}}$, is updated with the highest interferer for that $\mathrm{RB} \hat{k}_{h, x_{i}}$, with the corresponding ideal utility $u_{x_{i}, k_{h, x_{i}}}^{2}$ for the same RB and the user $x_{i}$ scheduled on that RB and with the difference between the ideal and measured SINR $\gamma_{x_{i}, k}^{2}-\gamma_{x_{i}, k}^{1}$. The columns of $\boldsymbol{U}^{\mathbf{1}}$, corresponding to the assigned set of resource blocks $\hat{k}_{h}$, are deleted and the process is repeated until all the resource blocks have been assigned. The $\mathrm{n}$ algorithm has been proven to be optimal in an assignment problem where the number of resource blocks is equal to the number of users [23]. In case this identity is not valid, the IHA provides a good suboptimal solution that trades off performance for lowered computational complexity when compared with traditional convex optimization techniques.

\subsubsection{Restriction negotiation}

We define as a cluster the group of cells interfering with each other. A cluster can then have variable size depending on the propagation conditions as shown in Figure 1 and how the users are distributed within each cell. If there are many users in the cell edge, these will witness higher interference coming from the surrounding cells. Each sector exchanges with all the other sectors in a cluster its restriction list $\mathbf{R}$. The entries of the restriction lists contain information on which RB $k$ each sector would like the interfering sectors to block, the ideal utility $u_{x_{i}, k}^{2}$ user $x_{i}$, scheduled on RB $k$, in sector $s$ would achieve and difference between the ideal and measured SINR $\gamma_{x_{i}, k}^{2}-\gamma_{x_{i}, k}^{1}$. From now on, we refer to utility $u_{x_{i}, k}^{2}$ as the utility the sector would achieve on RB $k: u_{s, k}^{2}$. Then each sector generates a cluster utility matrix $\mathbf{Z}$ in this manner: each row of $\mathbf{Z}$ represents each sector in the cluster. If RB $k$ is marked for restriction in the restriction list of sector $s$, then $z_{s, k}$ is equal to the utility $u_{x, k}^{2}$. If multiple sectors request the same $\mathrm{RB}$, then the utilities $u_{s, k}^{2}$ are organized in descending order. For each conflicting sector, a new column corresponding to the RB $k$ is generated to account for multiple RB assignments. Each entry of these columns is zero except for the utility of the sector. If two or more of these utilities are equal, they remain on the same column. The cluster utility is then at most $S \cdot S \cdot K$ large in the absolute worst case scenario where all sectors interfere with all other sectors on all the resource blocks. Each sector possesses its cluster utility matrix $\mathbf{Z}$ and proceeds in assigning resource blocks to each sector using the IHA described previously. The base station can use the same process in the 'restriction definition' as well as in the 'restriction negotiation'; thus, the complexity of the overall algorithm is just function of the size of $\mathbf{Z}$. Once all the resource blocks are assigned to the sectors, these represent the resources each sector needs to maximize the global cluster rate and are stored in the final assignment matrix A. Subsequently, the highest interferers present in the restriction list, corresponding to each entry in $\mathbf{A}$, have to be restricted.

Each macrocell sector, then, determines from its $\mathbf{A}$ and the restriction list which resource blocks it has to restrict and avoids transmission on those resources thus maximizing the capacity in its cluster. A picocell, on the other hand, makes use of the difference between the ideal SINR and the measured one $\gamma_{s, k}^{\text {dif }}=\gamma_{s, k}^{2}-\gamma_{s, k}^{1}$ exchanged by the interfered sector $s$. If RB $k$ is then scheduled to be restricted, the transmit power of the picocell, on that RB, is reduced by the same difference in SINRs:

$$
P_{k}=\max \left(P_{\max }-\gamma_{s, k}^{d i f}, 0\right),
$$

where $P_{\max }$ is the maximum transmit power per RB in $\mathrm{dB}$. In this way, if the SINR difference is lower than the maximum transmit power on the RB, the pico base station can re-use that RB for additional transmission; otherwise, it is restricted in the same way as for the macro base station.

\subsection{Femto interference management}

An independent algorithm at the femtocell side is proposed to limit the interference introduced by these unplanned and uncontrolled small cells. Since no fast backhaul communication between the tiers is assumed, each femto access point has to be able to determine whether it creates interference to macro cell users. In this work, femto base stations possess sensing capabilities and are able to overhear uplink communication between close by macro and pico users to their serving base stations. This assumption is highly advised in order to enable interference management and guarantee QoS; as in [16], the authors have shown that low-power sensing of LTE signals is possible, and if the femto base station is able to sense 
which RBs the interfered user is been assigned, then it can reduce power on those specific resources. The femto access point then determines the channel gain between itself and the macro or pico neighbouring base stations; it also determines the channel gains between the itself and the macro (or pico) users nearby. The femtocell is then able to use water-filling to reduce the transmit power on the RBs assigned to the macro (or pico) user.

\subsection{Notes on the iterative hungarian method}

The Hungarian algorithm was found to be an optimal solution method for the assignment problem for square matrices in strongly polynomial time. This methods remains optimal as long as the number of workers is identical to resources to be assigned. If this is not the same, as in the problem considered, the optimal solution cannot be found so readily. One either strives for optimality using algorithms for complete searches in combinatorial optimization, such as the branch-and-bound [25], or applies a sub-optimal solution, such as the iterative Hungarian algorithm. In [26], the authors have compared these two methods and have noted that the iterative Hungarian is able to reach a solution close to the optimal one found by the branch-and-bound (up to $96.5 \%$ of the optimal solution) while at a significantly reduced computational complexity (the iterative Hungarian converges in $3.8 \%$ of the time required by the optimal method). In [27], the authors compare the iterative Hungarian method with an optimal myopic search for dynamic spectrum access. They show that the former reaches solutions very close to the optimal method but the computational complexity reduced by 25 times. The iterative Hungarian algorithm is then able to reach a good sub-optimal solution at the fraction of an exhaustive search.

\section{$4 \mathrm{ICIC}$ results}

In this section, the simulation scenarios and performance results are presented and discussed. A downlink heterogeneous LTE network has been simulated with seven macrocells and five picocells placed around the central macrocell. A fixed number of ten randomly placed femtocells is used. Users are randomly scattered over the area and feature constant mobility for the whole duration of the simulations. Figure 2 presents the network under test, where the hexagons represent the sectors of the macrocells, the triangle represent the picocells and the squares represent the femtocells.

The network operates in full buffer, and full CSI accuracy is assumed. The throughput, the signal power received by each user and the behaviour of each cell are recorded and analysed. A mixed indoor-outdoor environment has been considered for the pathloss, with the parameters presented in [20]. For all the simulations, the internal cell resource allocation is performed with

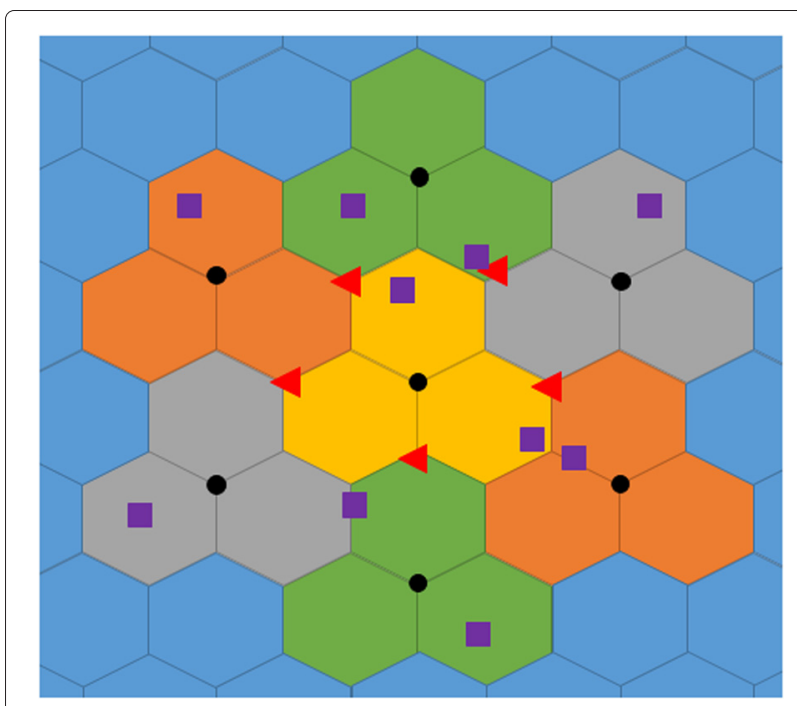

Figure 2 Network simulated.

the iterative Hungarian scheduler [28]. The simulation parameters are contained in Table 2.

\subsection{Results for homogeneous networks}

The average throughput of the macrocell users in a homogeneous LTE downlink network is analysed; the users are organized in percentiles, and these represent the portions of the users in the bottom \% of their categories: the fifth percentile users are the bottom $5 \%$ of the overall users, usually referred to as 'cell edge users'. The 100th percentile, on the other hand, represents the average cell rate. In these simulations, the proposed method is compared to the standard resource allocation (where no interference coordination is applied and only the internal allocation is performed) and the frequency reuse 3 where each cell uses one third of the available bandwidth, not overlapping with the neighbours, thus avoiding interference. The gains of the proposed method and the frequency reuse 3 technique over the non-coordinated solution are presented in Figure 3, where ICIC is the proposed method.

Figure 3 shows that since the proposed ICIC method is designed to target mainly the users which suffer from interference and are poorly served (lowest $5 \%$ to $10 \%$ ), it achieves high gain for low percentiles and no loss for high ones. On the contrary, the 'Reuse 3' method targets the whole bandwidth achieving high gain for the poorest served users but very high losses for all the other ones. The proposed method reaches, then, $86 \%$ of the gain of the other solution without any of its drawbacks.

\subsection{Results for heterogeneous networks}

Once the solution is extended to a heterogeneous network, with the parameters contained in Table 2, the 


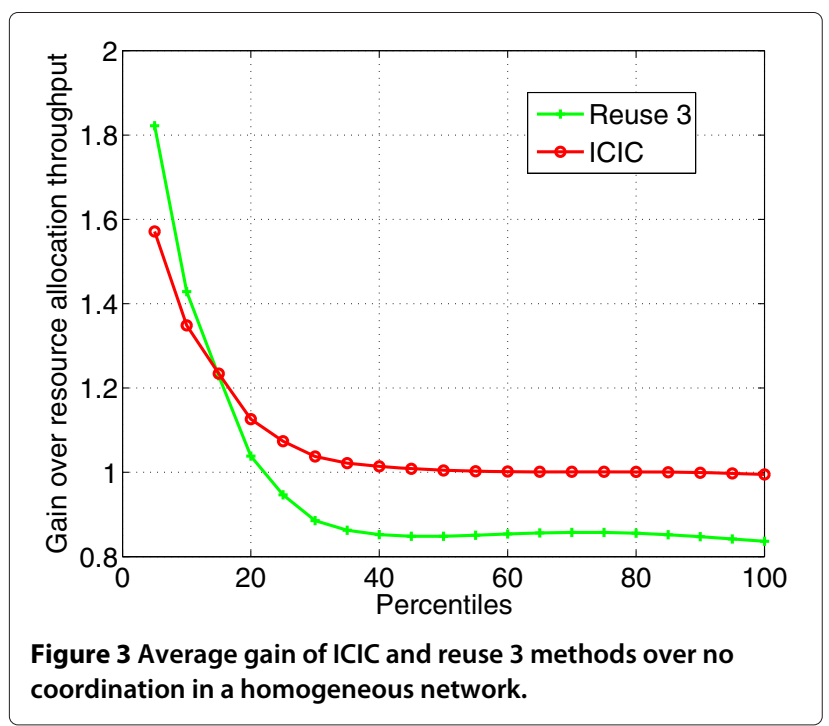

impact of the proposed method on macrocell users decreases as the macro users now witness additional interference coming form the pico- and femtocells and there are more cells which have to coordinate for the same amount of resources and the method can only address the highest interferer. Figure 4 presents the gains of the proposed solution for macro, pico and femto users (without any power control on pico or femtocells but only restricting the interfering RBs completely).

It is noticeable that both macro and pico user performances increase considerably, with a slight (4\%) loss for the highest performing macrocell users; this is due to the fact that picocells still interfere for those users but they do not get selected by the proposed method because of their good channel quality conditions (as the proposed solution only targets starved users). The combined gain for macro and pico users is shown in the black curve. On the other hand, femtocell users witness a lower performance due to the absence of communication between the tiers.

If power water-filling is allowed for pico and femto base stations are allowed in order to minimize losses, while still reducing overall interference the gains improve as shown in Figure 5. At the price of a very slight loss for macrocells users $(2 \%)$ due to the minimal increase in interference due to the non-complete restriction in transmission power, we see an increase in performance in both pico and femto users ( $10 \%$ and $4 \%$, respectively). The effect on the combined macrocell and picocell users shows that the picocell users tend to have higher throughput than the macrocell ones and thus the gains at lower percentiles are higher when compared to the previous implementation in Figure 4 but less than for just the picocell users. On the other hand, the complete gain shows also an improvement for the high percentiles, reducing the loss to $3 \%$. The results show that the proposed method works well and allows for a consistent increase in performance for the macro and pico users which outperforms traditional static solutions.

\section{Impact of CSI accuracy and feedback overhead}

In the previous sections, we have used state-of-the-art assumptions for our ICIC solution, i.e. that each base station has perfect information about the useful as well as

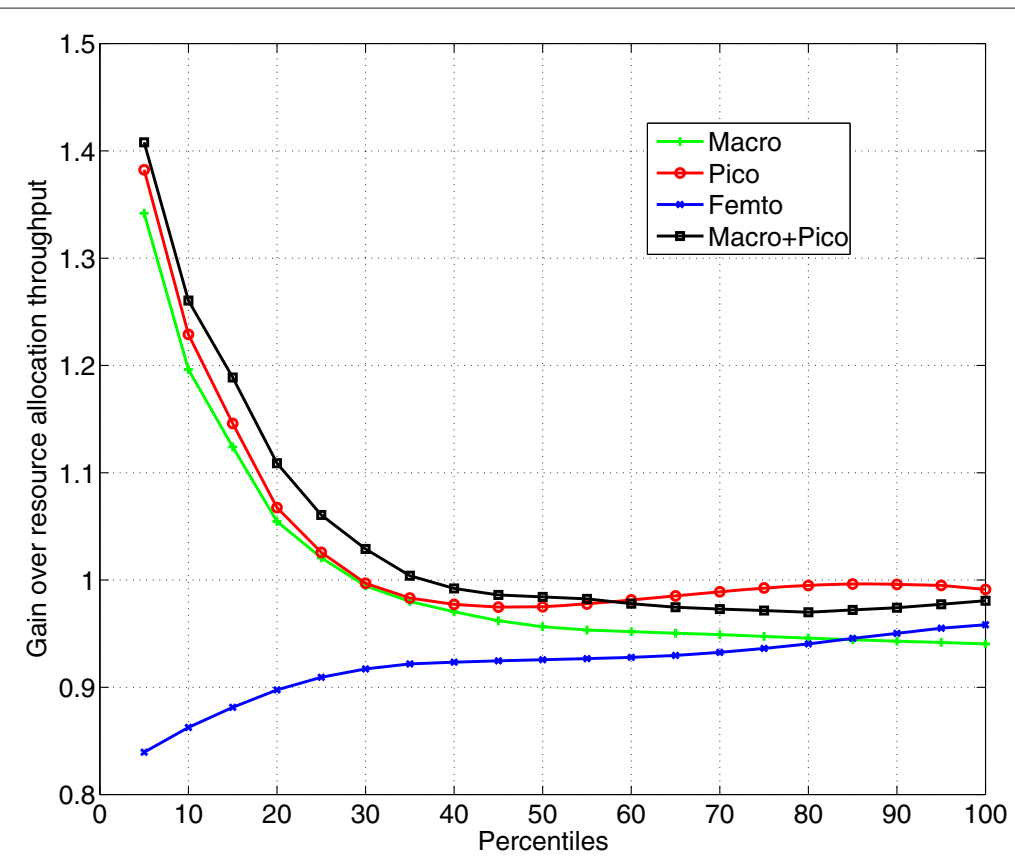

Figure 4 Gains of proposed method for macro, pico and femto users over resource allocation without any power control. 


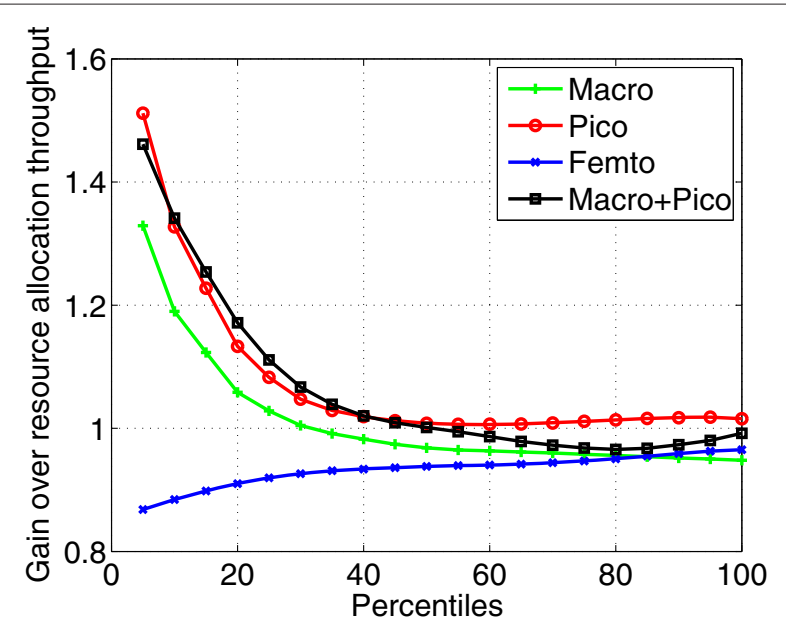

Figure 5 Gains of proposed method for macro, pico and femto users over resource allocation with power control.

interfering signal strength received by each of its users on each RB.

In reality, even though CQI could be measured on every $\mathrm{RB}$, the LTE standard allows only quantized information to be reported back to the base station to limit the amount of uplink resources necessary for signalling. Since ICIC techniques rely on the detailed information collected for each $\mathrm{RB}$, this limitation reduces the performance of any ICIC method.

Specifically, the LTE standard allows three different CSI quantization reporting methods to reduce the impact of signalling information on the uplink bandwidth:

- Wideband: each user transmits a single 4-bit CQI value for all the RBs in the bandwidth.

- Higher layer configured or subband level: the bandwidth is divided into $q$ subbands of $k$ consecutive RBs and each user feeds back to the base station one 4-bit wideband CQI and a 2-bit differential CQI for each subband. The value of $k$ is bandwidth dependent and is expressed in Table 3, where $N_{\mathrm{RB}}^{\mathrm{DL}}$ is total number of downlink RBs in the bandwidth (table 7.2.1-2 in [29]).

- User-selected or Best-M: each user selects $M$ preferred subbands of equal size $k$ and will transmit to the base station one 4-bit wideband CQI and a single 2-bit CQI value that reflects the channel quality only over the selected $M$ subbands.

Additionally, the user also reports the position of the selected subbands using $P_{\mathrm{FB}}$ bits, where $P_{\mathrm{FB}}$, as given in [29], is as follows:

$$
P_{\mathrm{FB}}=\left\lceil\log _{2}\left(\begin{array}{c}
N_{\mathrm{RB}}^{\mathrm{DL}} \\
M
\end{array}\right)\right\rceil .
$$

Table 3 Subband size $(k)$ vs. system bandwidth for subband level feedback

\begin{tabular}{lc}
\hline System bandwidth & Subband size \\
$\boldsymbol{N}_{\mathrm{RB}}^{\mathrm{DL}}$ & $(\boldsymbol{k})$ \\
\hline 6 to 7 & $\mathrm{NA}$ \\
8 to 10 & 4 \\
11 to 26 & 4 \\
27 to 63 & 6 \\
64 to 110 & 8 \\
\hline
\end{tabular}

The value of $M$ and the amount of RBs in each subband is given in Table 4 (table 7.2.1-5 in [29]).

In [19], we have introduced additional CSI quantizations based on the Best- $M$ method where the number of $M$ subbands is not fixed to 6 , and instead of a single CQI valid for all the subbands, one value per subband is used instead. This brings an increased amount of feedback while it allows the users to use a higher resolution of the best RBs using only wideband information for the others. We refer to this method as:

- Variable Best-M: In this work, the number of subbands varies between 1 and 5 and their size is bandwidth dependent and is chosen according to the values in Table 4.

In order for any ICIC mechanism to work, the users need to communicate some identifying information on the interferers to their serving base station. This procedure can be quite costly, in terms of bandwidth as a global cell identifier in LTE is set to be 28 bits long [30]. It is reasonable to assume that much fewer bits might be necessary for the system to operate as each base station can build a look-up table where the identities of the interfering neighbours are stored and each user simply feeds back the relative entries in the table. This additional overhead is not considered in this analysis as it is beyond the scope of this work to develop a compression scheme for the cell identifying information.

Table 4 Subband size $(k)$ and number of subbands $(M)$ vs. system bandwidth for user-selected feedback

\begin{tabular}{lcc}
\hline System bandwidth & Subband size & $\boldsymbol{M}$ \\
$\boldsymbol{N}_{\mathrm{RB}}^{\mathrm{DL}}$ & $(\boldsymbol{k})$ & \\
\hline 6 to 7 & $\mathrm{NA}$ & NA \\
8 to 10 & 2 & 1 \\
11 to 26 & 2 & 3 \\
27 to 63 & 3 & 5 \\
64 to 110 & 4 & 6 \\
\hline
\end{tabular}


Furthermore, the assumption that each user is capable of determining the identity of all the interfering base stations and to differentiate between the power received by them is a very common but weak one. In reality, a mobile user would not be capable of sensing the power of a signal with power very much lower than the wanted signal; an alternative solution is for a user to determine the identity of a wideband interferer by collecting the synchronization information over time.

Table 5 presents the throughput results for the 5 th, 50th and 95th percentiles normalized over the standard compliant 'subband level' feedback method. The different quantizations are applied to the useful signal in order to determine the user's channel quality and to the interference signals in order to determine the impact of the CSI quantization on the proposed ICIC method. In the table, the acronyms Full, SL, BM and $W$ refer to full resolution (per RB, no quantization), subband level, Best $M$ and wideband (only one CQI value is transmitted associated with the highest interferer), respectively. The variable Best- $M$ method is indicated instead with $B x$ where $x$ represents the amount of subbands used in the method. The throughput gains (or losses) are normalized over the subband level method as it represent the default method chosen in the LTE standard. Since acquiring information on the interference is more difficult than on the wanted signal, the CSI on the former is assumed have, generally, lower resolution than for the wanted signal. The last two columns in the table represent the cost of this signalling information on the uplink bandwidth in bits per single user and the amount of uplink bandwidth used by each user (with a $1 / 2$ code rate and a 16QAM modulation for a 20-MHz bandwidth).

The full resolution scenario is the one with the highest performance, but in practice, it cannot be used as it requires almost four times the amount of signalling information; this means that with only 20 users, an uplink 20 $\mathrm{MHz}$ uplink bandwidth would be exhausted [19].

Interestingly, methods such as variable Best- $M$ can successfully increase performance for low-performing users over the subband level state-of-the-art while decreasing the overall amount of signalling. This is due the fact that, with this method, users are able to allocate a high resolution to the subbands which present the highest quality and are thus most likely to be scheduled. A universal solution should take into account the practical limitations of the network and adapt the amount of signalling information necessary by each user accordingly to their relative performance.

\section{Conclusions}

In this paper, we propose a cooperative distributed heuristic algorithm for interference management in heterogeneous LTE networks. The proposed scheme efficiently allocates resources and reduces interference as it adapts to the network conditions. The method proposed here is scalable and adapts well to the dynamic behaviour of HetNets. We compared our scheme to well-known reference methods, and it delivers a consistent gain for the starved user's data rate in both homogeneous and heterogeneous networks. The scheme requires only communication between macro and pico base stations while the femtocells operate autonomously. It generates a gain of $45 \%$ for the combined macro and pico edge users at a very small cost for the cell centre lower than $4 \%$. It optimizes greatly picocell performance, with improvements of more than $50 \%$ at a small cost for femtocell users (15\%).

We have also studied the effects of quantizing CSI information on the proposed ICIC solution and shown that more resolution on the relevant $\mathrm{RBs}$ can increase

Table 5 CSI feedback cost

\begin{tabular}{|c|c|c|c|c|c|c|}
\hline \multicolumn{2}{|c|}{ Resolution } & \multicolumn{3}{|c|}{ Percentiles gain } & \multirow{2}{*}{$\begin{array}{c}\text { FB amount } \\
\text { (bits) }\end{array}$} & \multirow{2}{*}{$\begin{array}{c}\text { Uplink } \\
\text { percentage }\end{array}$} \\
\hline Useful signal & Interference & 5 th & 50th & 95th & & \\
\hline Full & Full & 8.65 & 2.21 & 1.77 & 408 & 4.9 \\
\hline Full & $\mathrm{SL}$ & 6.1 & 1.78 & 1.44 & 264 & 2.8 \\
\hline Full & W & 5.6 & 1.7 & 1.4 & 206 & 2.4 \\
\hline $\mathrm{SL}$ & $\mathrm{SL}$ & 1 & 1 & 1 & 120 & 0.71 \\
\hline$S L$ & W & 0.8 & 0.99 & 0.99 & 62 & 0.38 \\
\hline BM & $\mathrm{BM}$ & 0.94 & 1.03 & 1.01 & 148 & 0.85 \\
\hline $\mathrm{BM}$ & W & 0.86 & 1.01 & 1 & 76 & 0.46 \\
\hline B5 & W & 2.1 & 1.2 & 1.12 & 84 & 0.5 \\
\hline B4 & W & 2 & 1.12 & 1.05 & 70 & 0.43 \\
\hline B3 & W & 1.9 & 1.1 & 1 & 58 & 0.35 \\
\hline B2 & W & 1.65 & 0.9 & 0.9 & 44 & 0.27 \\
\hline B1 & W & 1.46 & 0.9 & 0.89 & 26 & 0.18 \\
\hline
\end{tabular}


performance while still maintaining the signalling information at levels lower than the current state of the art. The variable Best-M CSI quantization method improves the performance of both the resource allocation and the ICIC when compared to SoA solutions and encourages the network to use adaptive CSI quantizations to provide high-quality channel state information for the users who need it on the portions of the bandwidth more relevant for them.

\section{Competing interests}

The authors declare that they have no competing interests.

Received: 8 August 2014 Accepted: 9 February 2015

Published online: 14 March 2015

\section{References}

1. 3GPP, UTRA-UTRAN Long Term Evolution (LTE) and 3GPP System Architecture Evolution (SAE) (3rd Generation Partnership Project, May 2006). ftp://ftp.3gpp.org/Inbox/2008 web files/LTA Paper.pdf

2. $\mathrm{P}$ Godlewski, M Maqbool, M Coupechoux, J-M Kélif, in Proceedings of the 3rd International Conference on Performance Evaluation Methodologies and Tools. Analytical evaluation of various frequency reuse schemes in cellular OFDMA networks. ser. ValueTools '08 (ICST Institute for Computer Sciences, Social-Informatics and Telecommunications Engineering, ICST, Brussels, Belgium, 2008), pp. 32:1-32:10

3. R1-050507, Soft Frequency Reuse Scheme for UTRAN LTE. Huawei 3GPP TSG RAN WG1 Meeting no.41 Athens, Greece, May 2005

4. R Ghaffar, R Knopp, in Modeling and Optimization in Mobile, Ad Hoc and Wireless Networks (WiOpt) 2010 Proceedings of the 8th International Symposium on. Fractional frequency reuse and interference suppression for OFDMA networks, (2010), pp. 273-277

5. A Imran, M Imran, R Tafazolli, in Personal Indoor and Mobile Radio Communications (PIMRC) 2010 IEEE 21st International Symposium on. A novel self organizing framework for adaptive frequency reuse and deployment in future cellular networks, (2010), pp. 2354-2359

6. I Fraimis, V Papoutsis, S Kotsopoulos, in Global Telecommunications Conference (GLOBECOM 2010), 2010 IEEE. A decentralized subchanne allocation scheme with inter-cell interference coordination (ICIC) for multi-cell OFDMA systems, (2010), pp. 1-5

7. P Wang, C Liu, R Mathar, in Wireless Communication Systems (ISWCS) 2011 8th International Symposium on. Dynamic fractional frequency reused proportional fair in time and frequency scheduling in OFDMA networks, (2011), pp. 745-749

8. M Rahman, H Yanikomeroglu, in Vehicular Technology Conference, 2008. VTC Spring 2008. IEEE. Interference avoidance through dynamic downlink OFDMA subchannel allocation using intercell coordination, (2008), pp. $1630-1635$

9. Enhancing cell-edge performance: a downlink dynamic interference avoidance scheme with inter-cell coordination. Wireless Commun. IEEE Trans. 9(4), 1414-1425 (2010)

10. V Chandrasekhar, J Andrews, A Gatherer, Femtocell networks: a survey. Commun. Mag. IEEE. 46(9), 59-67 (2008)

11. A Galindo-Serrano, L Giupponi, Managing femto to macro interference without $\times 2$ interface support through POMDP. Mob. Netw. Appl. 17(5), 633-647 (2012). [Online]. Available: http://dx.doi.org/10.1007/s11036012-0394-y

12. D Lopez-Perez, A Valcarce, Roche de la G, J Zhang, OFDMA femtocells: a roadmap on interference avoidance. Commun. Mag. IEEE. 47(9), 41-48 (2009)

13. C Kosta, B Hunt, A Quddus, R Tafazolli, On interference avoidance through inter-cell interference coordination (ICIC) based on OFDMA mobile systems. Commun. Surv. Tutorials IEEE. 15(3), 973-995 (2013)

14. S Kaimaletu, R Krishnan, S Kalyani, N Akhtar, B Ramamurthi, in Communications (ICC) 2011 IEEE International Conference on. Cognitive interference management in heterogeneous femto-macro cell networks, (2011), pp. 1-6
15. A Chiumento, CDesset, S Pollin, L Van der Perre, R Lauwereins, in Workshop on Interference and Design Issues for Future Heterogeneous Networks, WCNC, 2014, IEEE. Scalable LTE interference mitigation solution for HetNet deployment, (2014)

16. A Chiumento, L Hollevoet, S Pollin, F Naessens, A Dejonghe, L Van der Perre, Diffs: a low power, multi-mode, multi-standard flexible digital front-end for sensing in future cognitive radios. J. Signal Process. Syst. 76(2), 109-120 (2014). [Online]. Available: http://dx.doi.org/10.1007/ s11265-014-0908-x

17. S Al-Rubaye, A Al-Dulaimi, J Cosmas, Cognitive femtocell. Vehicular Technol. Mag. IEEE. 6(1), 44-51 (2011)

18. S-Y Lien, K-C Chen, Y-C Liang, Y Lin, Cognitive radio resource management for future cellular networks. Wireless Commun. IEEE. 21(1), 70-79 (2014)

19. A Chiumento, C Desset, S Pollin, L Van der Perre, R Lauwereins, in Wireless Communications and Networking Conference (WCNC), 2014 IEEE. The value of feedback for LTE resource allocation, (2014)

20. J Ikuno, M Wrulich, M Rupp, in Vehicular Technology Conference (VTC 2010-Spring) 2010 IEEE 71 st. System level simulation of LTE networks, (2010), pp. 1-5

21. MB Stefania Sesia, Issam Toufik, LTE - The UMTS Long Term EvolutionFrom Theory to Practice. (John Wiley and Sons, Ltd., Chichester, 2009)

22. MR Garey, DS Johnson, Computers and intractability: a guide to the theory of NP-Completeness (Series of Books in the Mathematical Sciences). (W.H. Freeman \& Co Ltd., New York, 1979)

23. HW Kuhn, The hungarian method for the assignment problem. WNaval Res. Logistics Q. 2, 83-97 (1955)

24. P Kyösti, J Meinilä, L Hentilä, X Zhao, T Jämsä, C Schneider, M Narandzić, M Milojević, A Hong, J Ylitalo, V-M Holappa, M Alatossava, R Bultitude, Y de Jong, T Rautiainen, WINNER /l Channel Models, (2007). [Online]. Available http://www.ist-winner.org/deliverables.html Accessed on 6 Mar 2013

25. C Buchheim, A Caprara, A Lodi, in Integer Programming and Combinatorial Optimization. An effective branch-and-bound algorithm for convex quadratic integer programming. ser. Lecture Notes in Computer Science, vol. 6080, ed by. F Eisenbrand, F Shepherd (Springer Berlin, Heidelberg, 2010).pp. 285-298

26. J Oksanen, J Lunden, V Koivunen, in Acoustics, Speech and Signal Processing (ICASSP) 2012 IEEE International Conference on. Resource minimization driven spectrum sensing policy, (2012), pp. 3657-3660

27. M Bkassiny, S Jayaweera, Y Li, K Avery, in Vehicular Technology Conference (VTC Spring) 2011 IEEE 73rd. Optimal and low-complexity algorithms for dynamic spectrum access in centralized cognitive radio networks with fading channels, (2011), pp. 1-5

28. A Chiumento, S Pollin, C Desset, L Van der Perre, R Lauwereins, in Communications and Vehicular Technology in the Benelux (SCVT) 2012 IEEE 19th Symposium on. Analysis of power efficiency of schedulers in LTE, (2012), pp. 1-4

29. Third Generation Partnership Project, "Technical Specification Group Radio Access Network; Evolved Universal Terrestrial Radio Access (E-UTRA); Physical Layer procedures (Release 10)," 3GPP TS 36.213 V10.0.1 (2010). Available. http://www.3gpp.org/dynareport/36213.htm date of access: 6 Mar 2013

30. Third Generation Partnership Project, "Evolved Universal Terrestrial Radio Access Network (E-UTRAN); S1 Application Protocol (S1AP)", 3GPP TS 36.413 V10.0.1 (2011). Available. http://www.3gpp.org/dynareport/36413. htm date of access: 6 Mar 2013 